\title{
Total Thrombotic Occlusion of Aorta Distal To Inferior Mesenteric Artery
}

\author{
Fenil Raju Abraham ${ }^{1}$, Amit Mahajan ${ }^{2}$, Anil Luther ${ }^{3}$ \\ $\left({ }^{1}\right.$ Post Graduate Resident, ${ }^{2}$ Associate Professor, ${ }^{3}$ Professor) \\ Department of General Surgery, Christian Medical College and Hospital, Ludhiana- 141008, Punjab, India
}

\begin{abstract}
Complete aortic occlusion is a rare, and potentially fatal condition. it is usually seen in severe atherosclerotic disease. Here we present the case report of a 38 year old male, who presented with acute onset left leg pain and was found to have a complete thrombotic occlusion of the infra renal portion of abdominal aorta.
\end{abstract}

\section{Introduction}

Total occlusion of abdominal aorta is a rare and potentially catastrophic event, which needs prompt management. Else, it is associated with significant mortality and morbidity. The occlusion is usually attributed to two etiopathogenesis (1) Embolic occlusion and (2) Thrombotic occlusion. The terminal part of the abdominal aorta, distal to the renal arteries, especially the iliac bifurcation is the most frequent site of the occlusion. The case presented here, is that of a 38 year old male, with complete thrombotic occlusion involving the segment of abdominal aorta just distal to the origin of the inferior mesenteric artery, with diffuse extension of thrombus past the aortic bifurcation and involving the entire lower limbs.

\section{Case report}

A 38 year old male, a known case of peripheral vascular disease with previous history of right above knee amputation due to gangrene, presented to emergency department with complaints of claudicating pain in the left lower limb since last 2 weeks . Patient had history of smoking (15 packs / year). The claudicating pain had increased in intensity over the past week. There were no other known co-morbidities. His physical examination revealed non palpable femoral pulse on the left with weakly palpable femoral pulse on the right. The left lower extremity appeared cold with loss of hair, mild erythematous patches over the leg and cyanotic appearance of the foot. The remnant of the right lower extremity fared little better. He was planned for a CT angiogram, as a recent previous doppler showed severe peripheral arterial disease of the left side. The angiography (figures : 1- 4) detected a near total (95\%) luminal occlusion of the infra renal portion of the abdominal aorta just beyond the origin of the inferior mesenteric artery, with no appreciable contrast opacification of the bilateral common iliac, external iliac as well as the bilateral femorals ( common, superficial and deep). The distal vessels such as the popliteal, anterior and posterior tibial, dorsalis pedis and the plantar arteries showed total occlusion as well. A few collaterals were seen in bilateral thighs. The celiac axis, superior and inferior mesenteric arteries and the right renal artery were of normal calibre. Echocardiography and routine investigations were found to be normal. Patient was started on analgesics and injection heparin with regular monitoring of APTT values. A lumbar sympathectomy was then done to provide pain relief, as the analgesics were found to be inadequate. Patient symptomatically improved after that and was discharged in satisfactory condition with close follow up in the outpatient basis . 

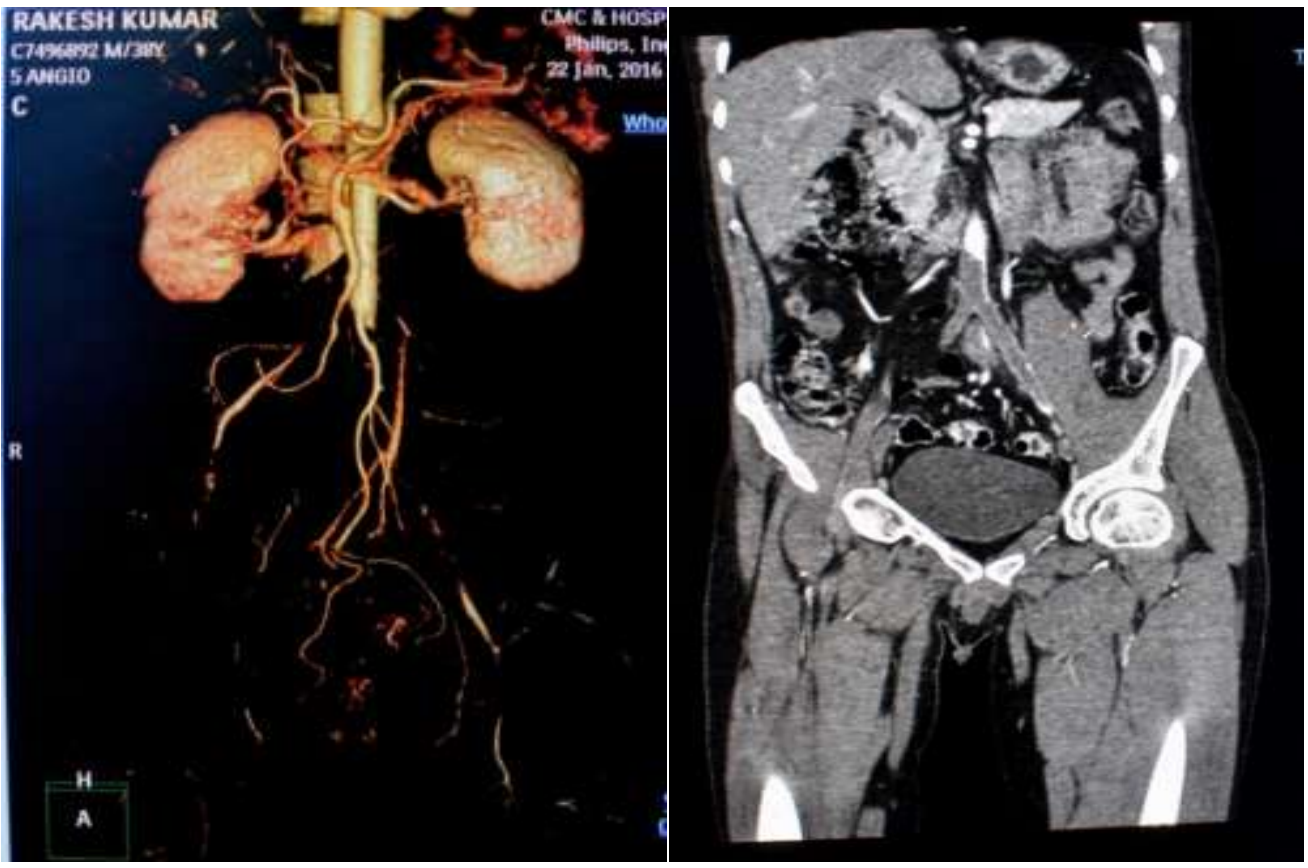

Figure 1: Three dimensional reconstruction of $\mathrm{CT}$ angiogram showing thrombotic occlusion below the inferior mesenteric arterv

Figure 2 : $\mathrm{CT}$ angiogram showing thrombus in abdominal aorta involving aortic bifurcation

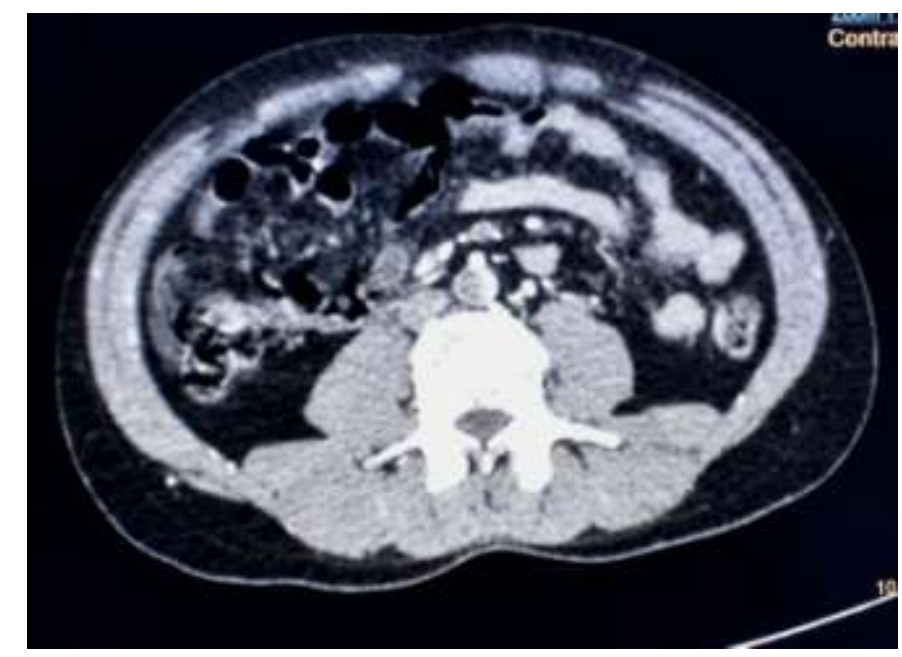

Figure 3: $\mathrm{CT}$ angiogram showing patent inferior mesenteric artery 


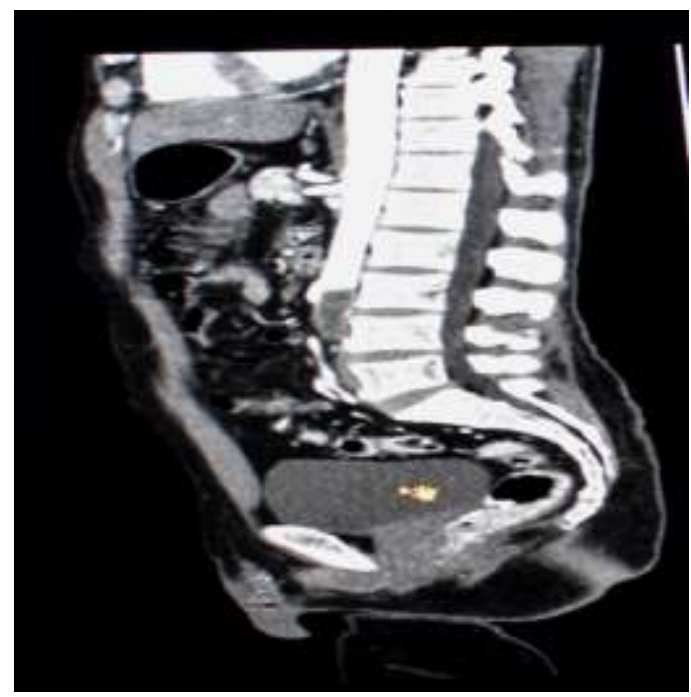

Figure 4 : CT angiogram showing aortic thrombus

\section{Discussion}

Acute abdominal aortic occlusion is a catastrophic event with a high rate of morbidity and mortality, that requires emergency intervention. The usual causes of the occlusion are either due to an embolism or due to a thrombus. The risk factors for embolism includes heart disease and female gender, while that of thrombosis includes smoking and diabetes. ${ }^{(1)}$ Pre-existing atherosclerosis with combined low flow state, is also a relatively frequent cause of acute aortic occlusion. ${ }^{(2)}$ The clinical presentation may vary from acute limb ischemia, neurological symptoms of lower extremities, abdominal symptoms and acute hypertension. The type of symptoms would depend upon the level of aortic occlusion. A simple and rapid test would be an ultrasound doppler to check the flow velocities.

Although aortography is considered the gold standard in these situations, but multi slice CT angiography would be an acceptable and accurate imaging modality in these conditions. Magnetic resonance angiography is a safer option, for preservation of kidney function. ${ }^{(3)}$

The diagnosis usually gets delayed due to the development of collaterals which maintain a basal perfusion and masks the acute ischemic events for a long time. A thrombotic cause may be considered in this patient who had a normal sinus rhythm and an echocardiogram showing no significant findings. ${ }^{(4)}$ In such patients, the treatment route is decided based on the clinical status and the co morbidities of the patient. Aortic reconstruction or femoral revascularisation via aorto-femoral bypass may be undertaken, especially in patients with a renal artery involvement. if no renal artery involvement is seen on angiogram, the initial treatment should be to re-establish inflow by retrograde femoral thromboembolectomy under local anaesthesia. ${ }^{(5)}$

\section{Conclusion}

Total thrombotic occlusion of the aorta in the infra mesenteric portion is a rare and potentially catastrophic entity, which needs rapid, accurate diagnosis and expedient management, to forestall rapidly increasing mortality and morbidity.

\section{References}

[1]. Dossa CD, Shepard AD, Reddy DJ, Jones CM, Elliott JP, Smith RF, et al. Acute aortic occlusion. A 40-year experience. Arch Surg 1994;129:603-7.

[2]. Buth J, Cuypers P. The diagnosis and treatment of acute aortic occlusions. Journal des maladies vasculaires 1996;21:133-5.

[3]. Singh A, Naqvi T, Marx MV, Rahimtoola SH. Acute thrombotic complete occlusion of the aorta: accurate, immediate clinical decision-making by echocardiography. Am J Med 2009; 122:e1

[4]. Zhang J, Duan ZQ, Wang CJ, Song QB, Luo YW, Xin SJ. Acute aortic occlusion as an unusual embolic complication of cardiac myxoma. Chin Med J (Engl) 2006;119:342-4

[5]. Webb KH, Jacocks MA. Acute aortic occlusion. Am J Surg 1988;155:405-7. 